\title{
BMJ Open Effects of e-aid cognitive behavioural therapy for insomnia (eCBTI) to prevent the transition from episodic insomnia to persistent insomnia: study protocol for a randomised controlled trial
}

\author{
Yuan Yang (D , ${ }^{1}$ Xian Luo, ${ }^{1}$ Dhirendra Paudel, ${ }^{1}$ Jihui Zhang, ${ }^{2}$ Shirley Xin Li, ${ }^{3}$ \\ Bin Zhang ${ }^{1}$
}

To cite: Yang Y, Luo X, Paudel D, et al. Effects of e-aid cognitive behavioural therapy for insomnia (eCBTI) to prevent the transition from episodic insomnia to persistent insomnia: study protocol for a randomised controlled trial. BMJ Open 2019;9:e033457. doi:10.1136/ bmjopen-2019-033457

- Prepublication history for this paper is available online. To view these files, please visit the journal online (http://dx.doi. org/10.1136/bmjopen-2019033457).

Received 06 August 2019 Revised 29 October 2019 Accepted 31 October 2019

Check for updates

(c) Author(s) (or their employer(s)) 2019. Re-use permitted under CC BY-NC. No commercial re-use. See rights and permissions. Published by BMJ.

${ }^{1}$ Department of Psychiatry, Southern Medical University Nanfang Hospital, Guangzhou, China

${ }^{2}$ Department of Psychiatry, The Chinese University of Hong Kong, Hong Kong, China

${ }^{3}$ Department of Psychology, University of Hong Kong, Hong Kong, China

Correspondence to

Dr Bin Zhang;

zhang73bin@hotmail.com

\section{ABSTRACT}

Introduction Previous evidence suggested that online self-guided sleep intervention is efficacious in improving treatment outcomes in patients with persistent insomnia. However, research on online sleep interventions targeting episodic insomnia has been scarce. This study aims to evaluate the effectiveness of brief e-aid cognitive behavioural therapy for insomnia (eCBTI) in preventing transition from episodic insomnia to persistent insomnia. Methods and analysis This is a pragmatic two-arm multicentre, randomised controlled trial comparing eCBTI with treatment as usual (TAU) in outpatients. Two hundred patients with episodic insomnia (as defined by DSM-5) will be recruited. Patients will be randomly assigned to receive 1 week eCBTI via a Smartphone application, or to receive TAU. Treatment effects will be assessed at 1 week and 3 months after intervention. The primary outcome of the study, whether the eCBTI program is sufficient in preventing transition from short-term to persistent insomnia, is measured by the Insomnia Severity Index. Secondary outcome measurements include the Dysfunctional Beliefs and Attitudes about Sleep Scale, Ford Insomnia Response to Stress Test, Sleep Hygiene and Practices Scale, Pre-sleep Arousal Scale and Epworth Sleepiness Scale. Additionally, the Hospital Anxiety and Depression Scale and the Short-Form 12-Item Health Survey will be used for measurement of mood symptoms and quality of life.

Ethics and dissemination Ethical approval for the study has been obtained from the Ethics Committee of Southern Medical University (reference number: NFEC-2017-131). The results of the investigation will be published in scientific papers. The data from the investigation will be made available online if necessary.

Trial registration NCT03302455 (clinicaltrials.gov). Date of registration: October 5, 2017.

\section{BACKGROUND}

\section{Insomnia disorder}

Insomnia is one of the most common sleep disorders. Most studies reported that $10 \%-15 \%$ of adults meet the clinical
Strengths and limitations of this study

- Participants will be randomly allocated using simple randomisation (computer-generated random numbers).

- An independent researcher will implement randomisation and treatment allocation through an automated online system.

- Onsite research staff will be blinded to the group assignment and study outcomes during the entire trial.

- Statistical analyses will be carried out by an independent researcher.

- Double-blinded study design cannot be achieved in the current study.

diagnostic criteria for insomnia disorder. ${ }^{1}$ On average, the economic burden of insomnia is US $\$ 5010$ per person per year, comparing with \$421 per year in an individual with good sleep, according to a study conducted in Quebec, Canada. ${ }^{2}$ In addition, indirect consequential impairment caused by insomnia (such as work-related injuries and sickness leave) is significantly greater than direct damage (such as cost of treatment of insomnia). Moreover, insomnia is a prodromal symptom and risk factor for the development and persistence of various physical and mental disorders. ${ }^{3-5}$

Based on the course of illness, insomnia is classified as 'persistent insomnia' (lasting 3 months or longer) or 'episodic insomnia' (lasting at least 1 month but less than 3 months). ${ }^{6}$ The diagnostic criteria for these two types of insomnia are similar, but persistent insomnia includes a minimum frequency of 'more than three nights per week' while episodic insomnia has no such criteria. ${ }^{6}$ It is worth mentioning that episodic insomnia is a common social phenomenon, and most people would have experienced it, especially 
in response to situational stress or rapid changes in the circadian rhythm. ${ }^{7}$ Episodic insomnia is often considered as a normal biopsychological response with no significant impairment; episodic insomnia attracts less research attention than persistent insomnia. ${ }^{8}$ Few longitudinal studies have investigated the natural course of insomnia. In the only two prospective studies conducted by Ellis et $a l$ specifically focusing on episodic insomnia, the annual prevalence of episodic insomnia was reported to be $36 \%$ and about $40 \%$ of the patients with episodic insomnia eventually developed persistent insomnia. ${ }^{9}{ }^{10}$ These two studies showed a high prevalence of episodic insomnia and a high susceptibility of developing long-term insomnia in those with episodic insomnia, which indicated that episodic insomnia could be a key transitional stage in the course of persistent insomnia. The findings suggested a need for developing timely, active intervention to prevent the conversion of episodic insomnia into persistent insomnia.

While sleep disturbances may gradually improve in some patients once the initial stressors are resolved, a portion of patients with episodic insomnia may eventually transit to developing persistent insomnia despite the resolution of the environmental stressors. ${ }^{6}{ }^{11}$ Due to the high recurrence rate of episodic insomnia, patients with episodic insomnia need to be actively treated. Furthermore, early psychobehavioural interventions and/or medication are important to prevent episodic insomnia from transition to persistent insomnia.

\section{Cognitive behavioural therapy for insomnia}

Currently, treatment for insomnia includes psychobehavioural intervention and/or medication. The psychobehavioural therapy mostly refers to cognitive behavioural therapy for insomnia (CBTI), which is a treatment approach for insomnia with a strong evidence base ${ }^{12} \mathrm{CBTI}$ aims to address the cognitive and behavioural factors that perpetuate insomnia, and consists of a constellation of treatment components, such as sleep hygiene education, relaxation therapy, stimulus control, sleep restriction and cognitive therapy. ${ }^{13}{ }^{14}$ In addition, CBTI may increase patients' self-efficacy and confidence to control their sleep problems and is currently suggested as the first-line treatment of insomnia in adults. ${ }^{15}$ However, the traditional CBTI program mainly focuses on the maintenance factors of insomnia, thus, it is mostly applied to treat persistent insomnia. ${ }^{15}$ A study with small a sample size $(\mathrm{n}=40)$ by Ellis $e$ al showed that a brief version of CBTI is effective in the treatment of acute insomnia. ${ }^{16}$ However, little is known about whether CBTI can prevent the transition of episodic insomnia to persistent insomnia. In addition, the dissemination of CBTI may be limited due to several obstacles. For example, the treatment procedure is complex, time-consuming and costly. ${ }^{17}$ It typically requires patients to travel to the hospital/clinic for face-to-face treatment, which may interfere with patients' routine work. In addition, CBTI is a specialised treatment approach which should be conducted by trained therapists; there may be significant variations between different therapists and clinical settings. Without proper guidance at home, patients may not be able to effectively apply the treatment strategies (eg, relaxation, stimulus control, sleep restriction), which in turn might hinder the resolution of insomnia. Additionally, CBTI requires patients to record their sleep pattern every night. This task might increase patients' anxiety and aggravate their insomnia symptoms. To address these challenges, internet-based CBTI has been developed and has been receiving widespread attention in recent years, as it makes the delivery of CBTI more efficient and flexible, and helps overcome the above shortcomings often associated with face-to-face treatment modality. ${ }^{18-20}$ Several e-aid CBTI (eCBTI) treatment tools have been made available in western countries (eg, Sleepio, SHUTi), and have shown similar efficacy as compared with standard CBTI. ${ }^{19}$ 21-24 However, further exploration and verification are still needed to examine the efficacy of eCBTI as treatment for episodic insomnia in the Chinese population.

\section{The current study}

In this study, we plan to establish a short-term eCBTI treatment program in Chinese to test whether eCBTI can reduce the transition from episodic insomnia to persistent insomnia. Moreover, we aim to investigate whether this program can improve sleep, anxiety, depression and quality of life in individuals with episodic insomnia.

The primary hypothesis for the trial is:

The eCBTI intervention can reduce transition of episodic insomnia to persistent insomnia.

The secondary hypotheses are:

1. The eCBTI intervention can improve sleep in patients with episodic insomnia.

2. The eCBTI intervention can improve depressive and anxiety symptoms in patients with episodic insomnia.

3. The eCBTI intervention can improve patients' overall health status and quality of life.

\section{METHODS/DESIGN \\ Research design}

This study is a parallel assignment, randomised controlled trial (RCT) to compare eCBTI intervention versus treatment as usual (TAU). The screening, assessments, allocation and intervention will all be carried out via a WeChat Mini Program (WMP) specially tailored for the trial. An information sheet will be provided online, and informed consent will be completed online before participation in the study. The research design is summarised in figure 1 .

\section{Participants}

We plan to recruit 200 participants diagnosed with episodic insomnia according to the sample size estimation. Participants will be recruited from sleep clinics in 31 hospital sites in Mainland China, and will be randomly allocated to two groups in a 1:1 ratio, namely, eCBTI $(\mathrm{n}=100,50 \%)$ or control group (TAU) $(\mathrm{n}=100,50 \%)$. The eCBTI group will receive eCBTI core treatment daily 
Complete online informed consent form

$\downarrow$

Online Constructed Questionnaires (socio-demographic information collected, and diagnostic interview based on DSM-5 for insomnia)

Inclusion criteria:

* A. Fulfilling the diagnostic criteria for episodic insomnia disorder according to DSM-5.

* B. 18 years of age or older.

* C. Compliant to the research program.

D. Own and know how to use smart gadgets (i.e. smartphones).

Exclusion criteria:

A. Diagnosis of definite and poor controlled physical diseases, menta disorders and/or sleep disorders requiring immediate attention.

B. Receiving some kind of psychological treatment for insomnia.

C. Shift workers, frequent night shift workers, frequent cross-time

fliers (such as international flight crevi).

Online baseline assessment

Self-rated questionnaires: Insomnia Severity Index (ISI), Dysfunctional Beliefs and Attitudes about Sleep (DBAS), Sleep Hygiene and Practices Scale (SHPS), Pre-sleep Arousal Scale (PSA), Ford Insomnia Response to Stress Test (FIRST), and Epworth Sleepiness Scale, (ESS). Additionally, participants are invited to complete the Hospital Anxiety and Depression Scale (HADS), and the Short-Form 12-Item Health Survey (SF-12). Research staff interview: MINI interview.

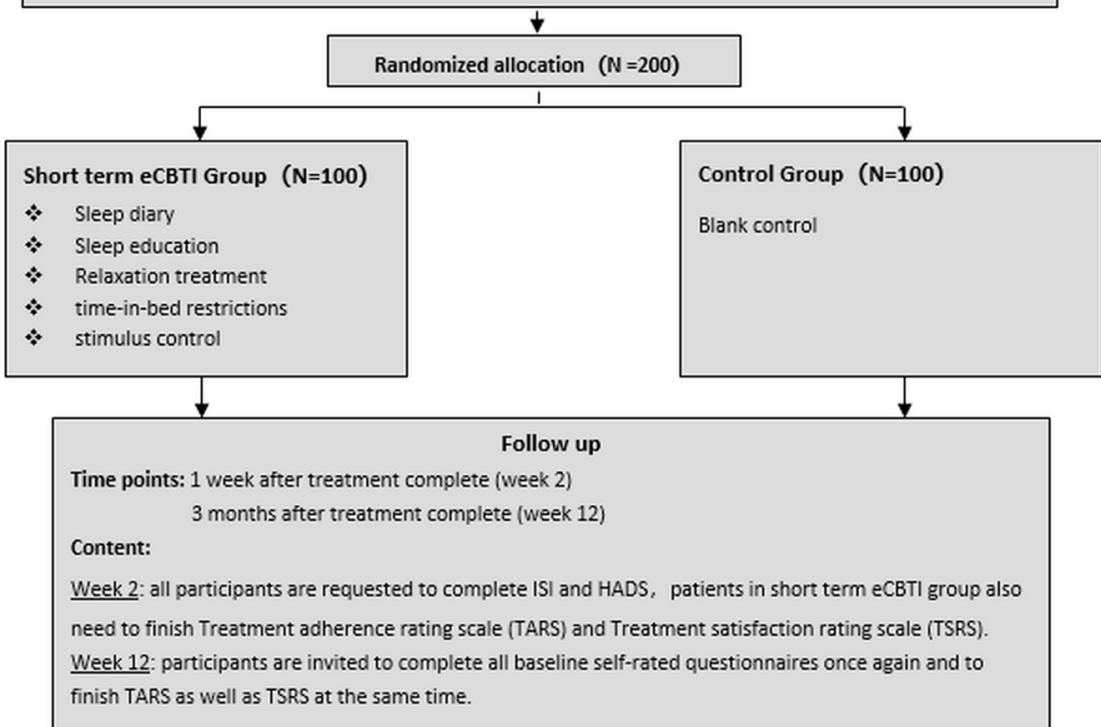

Figure 1 Recruitment flow chart. DSM-5, Diagnostic and Statistical Manual of Mental Disorders, Fifth Edition; eCBTI, e-aid cognitive behavioural therapy for insomnia; MINI, Mini International Neuropsychiatric Interview.

for 1 week in addition to TAU, while the control group will only receive TAU.

\section{Eligibility criteria}

To be included, participants must fulfil the following inclusion criteria:

- Meeting the diagnostic criteria for episodic insomnia according to DSM-5.

- Eighteen years of age or older.

- Being able to comply with the intervention.

- Provision of electronic informed consent.

- Own and know how to use smart gadgets (such as smartphones, tablets and computers).

Participants will be excluded if they meet the following exclusion criteria:

- Having a diagnosis of a significant untreated mental or medical illness (eg, consciousness disturbances, mania, acute phase of schizophrenia, major depressive disorder, etc).

- Have been receiving any kind of psychological treatment for insomnia in the past 6 months.

- Shift workers, frequent night shift workers, frequent cross-time fliers (eg, international flight crew, shifted nurse/health professionals).

To allow for greater generalisability, this study does not exclude patients with a stable condition of somatic disease, mental disorders (eg, depression in remission) and sleep disorders, or individuals receiving pharmacological treatments (eg, antihypertensive drugs, antidepressants and benzodiazepines).

\section{Randomisation}

This study is an RCT. Participants fulfilling the study criteria will be randomly allocated to either eCBTI 
group or control group using simple randomisation (computer-generated random numbers). ${ }^{25}$ An independent researcher from the IT department will implement randomisation and treatment allocation, which will be conducted through an automated online system. The research team will not be able to influence randomisation and will have no access to allocations.

\section{Blinding of assessment and analysis}

Onsite research staff will be blinded to the group assignment and study hypotheses of the trial. The independent researcher from the IT department who carries out the randomisation and allocation procedure will be blinded to the study protocol. Participants could not be blinded to treatment allocation as participants in the blank control group only received TAU. The research team will have limited contact with both IT staff and study participants, therefore, will not be able to bias the allocation or the assessments. Statistical analyses will be carried out by an independent researcher from the Southern Medical University who is not involved with the randomisation and assessment procedures.

\section{Assessment points}

Assessments will be conducted at week 0 (baseline), week 2 (1 week post-treatment) and week 12 (3 months after intervention). In consideration of ethical matters, participants in the control group will be offered eCBTI at week 12.

\section{Planned intervention}

After baseline assessment, participants will be randomly assigned to receive 1 week eCBTI or to receive TAU. eCBTI will be delivered using WMP. The program and all tools can be accessed using the WeChat app of any smartphone. With assistance from several professional IT staff and clinical psychologists, the eCBTI intervention program has been well developed and tested before the start of our current study. Participants in the eCBTI group will receive core sessions daily for 1 week. Participants will be provided with individualised treatment with the behavioural components (eg, stimulus control, sleep restriction) according to their sleep pattern in the past 2 weeks prior to the treatment session, as well as the cognitive components (eg, cognitive restructuring, paradoxical intention). Treatment elements also include daily sleep diary, relaxation audios (eg, body scan, breathing exercise) and sleep hygiene education. The treatment content is designed based on CBTI guidelines. ${ }^{13} 2627$

\section{Assessment of safety}

CBTI is a cognitive behavioural therapy and its risk of severe adverse events is low. Previous studies have indicated that online CBTI is rather safe and did not report any adverse outcomes. ${ }^{28}$ In the current study, participant's insomnia severity will be monitored by subjective measurements during treatment and at 3 months follow-up. Additionally, psychiatrists will be involved to assess the participant's suicidal ideation and professional intervention will be provided to those in need. Participants will be allowed to attend their usual clinical follow-up in the clinic and concurrently receive routine treatments for their clinical conditions, where needed. Any participant who reports worse insomnia symptoms after the completion of the intervention will be introduced to receive standard treatment for insomnia (medication and/or non-pharmaceutical treatment).

\section{Outcome measures}

Participants will receive a WeChat notification to complete the assessments online. At all times, all the assessments will be consistent across participants. If participants do not complete the questionnaire within 2 days, they will receive a reminder message. At baseline, demographics and related clinical data will be collected. Descriptive data on lifestyle practices such as tea and coffee consumption, smoking and alcohol use will also be recorded.

\section{Primary outcome measures}

The change in insomnia symptoms will be measured by the Insomnia Severity Index (ISI), ${ }^{29}{ }^{30}$ which assesses the severity, nature and impact of insomnia. It is a 7-item self-report measure, ranging from 0 (no problem) to 4 (very severe problem). The resulting sum score of the ISI ranges from 0 to 28. This outcome will be measured again at week 2 ( 1 week after treatment is complete) and week 12 (3 months follow-up) in both the eCBTI and control groups.

Another primary outcome measure is the Ford Insomnia Response to Stress Test, ${ }^{31}$ a measure to identify sleep disturbance and predisposition to persistent insomnia. Scores on this 9-item self-report questionnaire range from 9 to 36 .

\section{Secondary outcome measures}

The subjects' general sleep hygiene and practices will be measured with the Sleep Hygiene and Practices Scale. ${ }^{32}$ The Dysfunctional Beliefs and Attitudes about Sleep Scale ${ }^{33}$ will be used to measure sleep-related beliefs, potential treatments, expectations and attitudes towards causes. Problems of sleep initiation will be assessed with the PreSleep Arousal Scale. ${ }^{34}$ We will also assess the patient's chronotype using the 5-item Morningness-Eveningness Questionnaire, ${ }^{35}$ the generic health outcomes from the patient's perspective using the Short Form 12-Item Health Survey, ${ }^{36}$ and anxiety and depression levels using the Hospital Anxiety and Depression Scale. ${ }^{38}$ Participants in the treatment group will be also asked to complete a selfreported questionnaire to assess their treatment adherence and perceived helpfulness using the Treatment Adherence Scale. ${ }^{39}$ Participants' satisfaction with the treatment will be measured using the Treatment Satisfaction Scale. ${ }^{40}$

\section{Sample size estimation}

Previous studies have indicated that approximately $40 \%$ of patients with episodic insomnia transit to persistent insomnia. ${ }^{9}$ Based on our previous clinical experience, 
we anticipate that more than $70 \%$ of the subjects will have been retained at 12 weeks follow-up. In order to meet the 95\% CIs with 35\%-49\% requirement, the current project needs 200 cases of episodic insomnia. This sample size ensures the the statistical effect is greater than 0.8 in continuous data of a small sample (Cohen $\mathrm{d}=0.30$ ), and also ensures that the $\mathrm{OR}$ of dichotomous variables is greater than $1.50(\mathrm{p}>0.05)$.

\section{Statistical analysis}

Intention to treat (ITT) ${ }^{41}$ will be used for the main efficacy analysis and per protocol (PP) for the consistency test. The ITT group consists of all participants who have undergone at least 1 week of treatment and evaluation. The PP group refers to all ITT subjects who have not experienced significant program deviation or violation. In the ITT analysis, the last observation carried forward method will be used to analyse any missing therapeutic data.

Mean with SD for continuous variables, and frequency with a percentage for categorical variables will be reported. Independent t-test and non-parametrical analyses, where appropriate, will be applied to compare the differences between the two groups. Repeated measurement analysis will be used to compare the changes of symptoms (eg, ISI Score) following treatment and at 3 months follow-up. The clinical outcome of categorical variables will be computed using survival analysis or $\chi^{2}$ test, such as the appearance of suicidal ideation (as measured by the 'Yes/ No' question). Statistical analyses will be conducted using SPSS analytics software V.22.0. Alpha will be set at 0.05 for all tests (two-sided).

\section{Ethics and dissemination}

An electronic informed consent form will be signed by all participants. Participants will be assured that the participation is completely voluntary, and their information will be kept confidential. The research team will have access to the final data set and make the final decision to terminate the trial.

\section{Patient and public involvement}

Patients or the public were not involved in the design, conduct, reporting or dissemination of our research.

\section{DISCUSSION}

Episodic insomnia is a common phenomenon and might be a significant contributory causal factor in the transition from episodic insomnia to persistent insomnia. eCBTI is more efficient, flexible and time-saving compared with traditional face-to-face CBTI, and may also help to improve an individual's mood disturbance (eg, anxiety and depressive symptoms) as well as quality of life. The main aim of the current trial is to investigate whether a brief eCBTI program is effective in preventing the transition from acute insomnia to persistent insomnia. A previous placebo-controlled RCT demonstrated that online CBTI yields an effect size comparable to that of a traditionally delivered face-to-face therapy. ${ }^{28}$ Furthermore, a previous RCT has provided some preliminary support for the efficacy of treating acute insomnia with a test of single-shot CBTI. ${ }^{16}$ The study design benefits from being administered online with a smartphone app which is used widely in China, allowing us to recruit adequate research participants and limit researcher bias during the conduct of the study. Nonetheless, the study will test the key hypothesis that episodic insomnia is a contributory causal factor or the natural course in the occurrence of persistent insomnia.

\section{Limitation}

First, a double-blinded study design cannot be achieved in the current study, and only the onsite research staff are blinded to the group assignment. Second, as the sample size of this study is relatively small, the results might thus not be representative of the general population. Third, since sleep measurements in our study are mainly based on self-reports, apart from subjective sleep measurements, objective measurements, such as actigraphy and polysomnography would be beneficial.

\section{Trial status}

Recruitment began in October 2017. It is anticipated that recruitment will be complete by October 2019 , and that trial results will be available in 2020 .

Contributors Study design: BZ. Drafting of the manuscript: YY, XL and DP. Critical revision of the manuscript: JZ, SXL and BZ. Approval of the final version for publication: all coauthors.

Funding This study is funded by the President Foundation of Nanfang Hospital, Southern Medical University (2017L001), Key Item of Guangzhou bureau of education (2019KC106), Innovation Item of Guangdong Provincial Department of Education (2018A043442), and Project of Guangzhou Philosophy and Society Development (2018GZGJ58).

Competing interests None declared.

Patient consent for publication Not required.

Provenance and peer review Not commissioned; externally peer reviewed.

Open access This is an open access article distributed in accordance with the Creative Commons Attribution Non Commercial (CC BY-NC 4.0) license, which permits others to distribute, remix, adapt, build upon this work non-commercially, and license their derivative works on different terms, provided the original work is properly cited, appropriate credit is given, any changes made indicated, and the use is non-commercial. See: http://creativecommons.org/licenses/by-nc/4.0/.

\section{ORCID iD}

Yuan Yang http://orcid.org/0000-0002-2914-9832

\section{REFERENCES}

1 Zhang B, Wing Y-K. Sex differences in insomnia: a meta-analysis. Sleep 2006;29:85-93.

2 Daley M, Morin CM, LeBlanc M, et al. The economic burden of insomnia: direct and indirect costs for individuals with insomnia syndrome, insomnia symptoms, and good sleepers. Sleep 2009;32:55-64.

3 Li SX, Zhang B, Li AM, et al. Prevalence and correlates of frequent nightmares: a community-based 2-phase study. Sleep 2010;33:774-80.

4 Baglioni C, Spiegelhalder K, Feige B, et al. Sleep, depression and insomnia - a vicious circle? Curr Psychiatry Reviews 2014;10:202-13. 
5 Fernandez-Mendoza J, Vgontzas AN. Insomnia and its impact on physical and mental health. Curr Psychiatry Rep 2013;15:418.

6 American Psychiatric Association. Diagnostic and statistical manual of mental disorders. 5th edn. Washington, DC: APA, 2013.

7 House PsMP. Guidelines for diagnosis and treatment of insomnia in People's Republic of China. China: People's Medical Publishing House, 2016.

8 Ellis JG, Gehrman P, Espie CA, et al. Acute insomnia: current conceptualizations and future directions. Sleep Med Rev 2012;16:5-14.

9 Ellis JG, Perlis ML, Neale LF, et al. The natural history of insomnia: focus on prevalence and incidence of acute insomnia. J Psychiatr Res 2012;46:1278-85.

10 Ellis JG, Perlis ML, Bastien CH, et al. The natural history of insomnia: acute insomnia and first-onset depression. Sleep 2014;37:97-106.

11 American Academy of Sleep, M. International classification of sleep disorders, 2014.

12 Drerup M. Insomnia, cognitive behavioral treatment of, in encyclopedia of the neurological sciences. Oxford: Academic Press, 2014: 708-10.

13 House PsMP. Cognitive behavioral treatment of insomnia - a sessionby-session guide. China: People's Medical Publishing House, 2012.

14 Sivertsen B. Cognitive behavioural therapy for insomnia. 2009: 2, 61-34.

15 Smith MT, Huang MI, Manber R. Cognitive behavior therapy for chronic insomnia occurring within the context of medical and psychiatric disorders. Clin Psychol Rev 2005;25:559-92.

16 Ellis JG, Cushing T, Germain A. Treating acute insomnia: a randomized controlled trial of a "single-shot" of cognitive behavioral therapy for insomnia. Sleep 2015;38:971-8.

17 Perils ML, Smith MT. How can we make CBT-I and other BSM services widely available? J Clin Sleep Med 2008;4:11-13.

18 Espie CA. "Stepped care": a health technology solution for delivering cognitive behavioral therapy as a first line insomnia treatment. Sleep 2009;32:1549-58.

19 Cheng SK, Dizon J. Computerised cognitive behavioural therapy for insomnia: a systematic review and meta-analysis. Psychother Psychosom 2012;81:206-16.

20 Ho FY, Yeung WF, Chung K-F. Self-Help cognitive-behavioral therapy for insomnia (CBT-I): a systematic review of randomized controlled trials. 15th Nordic Conference, Copenhagen, Denmark, 2015.

21 van Straten A, Cuijpers P. Self-help therapy for insomnia: a metaanalysis. Sleep Med Rev 2009;13:61-71.

22 Ritterband LM, Bailey ET, Thorndike FP, et al. Initial evaluation of an Internet intervention to improve the sleep of cancer survivors with insomnia. Psychooncology 2012;21:695-705.

23 Espie CA, Kyle SD, Miller CB, et al. Attribution, cognition and psychopathology in persistent insomnia disorder: outcome and mediation analysis from a randomized placebo-controlled trial of online cognitive behavioural therapy. Sleep Med 2014;15:913-7.

24 Espie CA, Luik Al, Cape J, et al. Digital cognitive behavioural therapy for insomnia versus sleep hygiene education: the impact of improved sleep on functional health, quality of life and psychological well-being. study protocol for a randomised controlled trial. Trials 2016;17:257
25 Hewitt CE, Torgerson DJ. Is restricted randomisation necessary? BMJ 2006;332:1506-8.

26 Espie CA, Inglis SJ, Tessier S, et al. The clinical effectiveness of cognitive behaviour therapy for chronic insomnia: implementation and evaluation of a sleep clinic in general medical practice. Behav Res Ther 2001;39:45-60.

27 Espie CA, MacMahon KMA, Kelly $\mathrm{H}-\mathrm{L}$, et al. Randomized clinical effectiveness trial of nurse-administered small-group cognitive behavior therapy for persistent insomnia in general practice. Sleep 2007;30:574-84.

28 Espie CA, Kyle SD, Williams C, et al. A randomized, placebocontrolled trial of online cognitive behavioral therapy for chronic insomnia disorder delivered via an automated media-rich web application. Sleep 2012;35:769-81.

29 Morin CM, Belleville G, Bélanger L, et al. The insomnia severity index: psychometric indicators to detect insomnia cases and evaluate treatment response. Sleep 2011;34:601-8.

30 Shapour BA, Gang CK. Reliability and validity of the Chinese translation of insomnia severity index and comparison with Pittsburgh sleep quality index. Malaysian J Psychiatry 2013;22:1-7.

31 Gao CYet al. Initial application of Chinese version of Ford insomnia response to stress test scale. J Clin Psychiatry 2014:305-7.

32 Yang C-M, Lin S-C, Hsu S-C, et al. Maladaptive sleep hygiene practices in good sleepers and patients with insomnia. $J$ Health Psychol 2010;15:147-55.

33 Espie CA, Inglis SJ, Harvey L, et al. Insomniacs' attributions. psychometric properties of the dysfunctional beliefs and attitudes about sleep scale and the sleep disturbance questionnaire. $J$ Psychosom Res 2000;48:141-8.

34 Nicassio PM, Mendlowitz DR, Fussell JJ, et al. The phenomenology of the pre-sleep state: the development of the pre-sleep arousal scale. Behav Res Ther 1985;23:263-71.

35 Wei-Xia Llet al. Validity and reliability of the Chinese version of Morningness/Eveningness Questionnaire-5 items(MEQ-5) in students of technical schools. Chinese Mental Health Journal 2016;30:406-12.

36 Ware J, Kosinski M, Keller SD. A 12-Item short-form health survey: construction of scales and preliminary tests of reliability and validity. Med Care 1996;34:220-33.

37 Shou J, Ren L, Wang H, et al. Reliability and validity of 12-Item short-form health survey (SF-12) for the health status of Chinese community elderly population in Xujiahui district of Shanghai. Aging Clin Exp Res 2016;28:339-46.

38 Zigmond AS, Snaith RP. The hospital anxiety and depression scale. Acta Psychiatr Scand 1983;67:361-70.

39 Milosevic I, Levy HC, Alcolado GM, et al. The treatment Acceptability/Adherence scale: moving beyond the assessment of treatment effectiveness. Cogn Behav Ther 2015;44:456-69.

40 DiBenedetti DB, Gondek K, Sagnier P-P, et al. The treatment satisfaction scale: a multidimensional instrument for the assessment of treatment satisfaction for erectile dysfunction patients and their partners. Eur Urol 2005;48:503-11.

41 Alshurafa M, Briel M, Akl EA, et al. Inconsistent definitions for intention-to-treat in relation to missing outcome data: systematic review of the methods literature. PLoS One 2012;7:e49163. 